\title{
1 The generation of a Bcl11a lineage tracing mouse model
}

2 Sara Pensa ${ }^{1,2}$, Pentao Liu ${ }^{3,4}$, Walid T. Khaled ${ }^{1,2^{*}}$

1. University of Cambridge, Department of Pharmacology, Cambridge, CB2 1PD, UK

2. Wellcome-MRC Cambridge Stem Cell Institute, Cambridge, CB2 OAW, UK

3. Wellcome Trust Sanger Institute, Wellcome Genome Campus, Hinxton, Cambridge CB10 1HH, UK

4. Present address: School of Biomedical Sciences, University of Hong Kong, Hong Kong

* Correspondence should be directed to wtk22@cam.ac.uk

\section{Abstract}

The transcription factor B-cell lymphoma/leukaemia 11A (BCL11A) has essential functions in physiological processes as well as haematological and solid malignancies, however, its contribution to tissue development and tumour progression is poorly understood. Here we show the generation of a Bcl11a lineage tracing mouse model to allow for in vivo tracking of Bc/11a-expressing cells and their progeny. We validate the model in the mammary gland by using flow cytometry and whole-tissue 3D imaging to locate labelled cells after induction of tracing in early development. We show that $\mathrm{Bc} / 11 \mathrm{a}$ is predominantly expressed in longlived luminal progenitors which populate mammary alveoli upon pregnancy, confirming bona fide labelling of $\mathrm{Bcl11a}$ cells. The $\mathrm{Bcl11a}$ lineage tracing mouse model therefore provides a powerful resource to study Bcl11a cells in development, homeostasis, and cancer.

\section{Introduction}

The transcription factor $B$-cell lymphoma/leukaemia 11A $(B C L 11 A)$ is a zinc finger protein mainly expressed in the brain and hematopoietic cell lineages ${ }^{1,2}$. BCL11A is part of the ATP-dependent chromatin remodelling mammalian SWI/SNF complex ${ }^{3}$, which enables nucleosome restructuring, ensuring DNA is accessible for transcription replication and DNA repair ${ }^{4}$. 
$30 B C L 11 A$ is essential for B-cell development and plays a crucial role in the foetal to adult

31 haemoglobin switch ${ }^{5-7}$. Evidence is also accumulating of its role in various other

32 physiological processes, including a developmental role in the skin and in determining

33 dendritic cell fate ${ }^{8,9}$. It is also implicated in the $2 \mathrm{p} 15-\mathrm{p} 16.1$ microdeletion syndrome,

34 characterised by intellectual disability, Type II diabetes and $\beta$-hemoglobinopathies,

35 particularly sickle cell disease (SCD) ${ }^{1}$. Targeting $B C L 11 a$ has been implemented to

36 regulate foetal haemoglobin $(\mathrm{HbF})$ levels in erythrocytes in a transgenic humanized sickle

37 cell mouse model and in SCD patients, thus providing preliminary evidence of the

38 therapeutic effects of such a strategy for $\beta$-hemoglobinopathies ${ }^{10,11}$.

39 In addition, BCL11A is highly expressed in some haematological malignancies and malignant solid tumours and is associated with poor clinical prognosis ${ }^{1}$.We have previously shown that $B C L 11 A$ is a triple negative breast cancer (TNBC) gene ${ }^{12}$. BCL11A is overexpressed in TNBC including basal-like breast cancer (BLBC), and its genomic locus

43 is amplified in up to $38 \%$ of BLBC. BCL11A overexpression in cell lines promotes tumour 44 formation whereas its knockdown supresses tumour formation in xenograft models. Importantly, Bcl11a deletion severely decreases tumour formation in a mouse model of $\mathrm{TNBC}^{12}$.

We have also characterised BCL11A role as a lung squamous carcinoma (LUSC) oncogene $^{13}$. We showed that $B C L 11 A$ is upregulated in LUSC but not in lung adenocarcinomas and that knockdown of BCL11A in LUSC cell lines abolishes xenograft tumour growth and its overexpression in vivo leads to lung airway hyperplasia and the development of reserve cell hyperplastic lesions ${ }^{13}$. At the molecular level we found $B C L 11 A$ 52 to be a target of SOX2, a key driver of $\operatorname{LUSC}^{14}$, and we showed that it was required for the 53 oncogenic role of SOX2 in LUSC ${ }^{13}$. Furthermore, we showed that BCL11A and SOX2 54 interact at the protein level and that together they co-regulate the expression of several 
transcription factors, including STED8, and inhibition of STED8 selectively affects LUSC growth $^{13}$.

57 Despite its implication in various pathophysiologies, BCL11A contribution to tissue development and tumour initiation is poorly characterised. Lineage tracing is the most widely used technique to track stem and progenitor cells and their progeny in vivo and to explore the contribution of these cells to development, homeostasis, and cancer ${ }^{15}$. The fates of individual cells can be traced using both spatial and temporal control. Well characterised gene promoters drive expression in the cell types of interest, while inducible systems allow for temporal control. The main inducible systems used for stem cell biology are a tamoxifen-regulatable version of the Cre recombinase that is engineered behind an endogenous or transgenic promoter and a tetracycline-responsive reverse transactivator (rtTA) linked to a cell-specific promoter, incorporating a separate TetO-Cre allele.

Considering BCL11A important functions in tissue physiology, as well as its widespread implication in malignancies, we sought to generate a lineage tracing mouse model implementing the tamoxifen-inducible Cre as a strategy to trace Bcl11a-expressing cells fate, and validated the labelling strategy in mammary epithelial cells.

\section{Results}

73 The Bcl11a lineage tracing mouse model was generated by knocking-in an IRES-CreERT2 cassette into the $3^{`}$ UTR region of $B c / 11 a$, which we hereafter refer to as $B c / 11 a^{\text {CreERT2 }}$ (Figure 1A). The Bc/11aCreERT2 allele was generated by introducing an IRES-CreERT2polyA-FRT-PGK-E7-Neo-polyA-FRT cassette into the 3'UTR of Bcl11a locus. Gene targeting was carried out in AB2.2 cells (129 background). Correct targeting was confirmed 
were then crossed to wildtype mice (C57/B6) and germline transmission was confirmed (Figure 1B). These mice allowed us to perform lineage tracing when crossed to the Rosa26-LSL-tdTomato reporter mice (tdTom) to generate double transgenic mice (Figure 1C). Tamoxifen mediated CreERT2 activation induced tdTomato expression in $\mathrm{Bc} / 11 \mathrm{a}$ expressing cells and any of their future progeny. We have shown before that $B c / 11 a$ is expressed predominantly in luminal progenitor cells and, in smaller proportion, in basal cells and differentiated luminal cells in the adult mammary gland ${ }^{12}$. To confirm that tamoxifen administration leads to true labelling of Bcl11a expressing cells, we checked If this was the case in the mammary gland by inducing lineage tracing in the adult with 3 injections on 3 consecutive days of tamoxifen ( $1 \mathrm{mg}$ per injection). Mammary tissues were collected at 1 day ( 1 day p.i.) or 5 days (5 days p.i.) post injection and RNA was extracted from epithelial cells. At 1 day p.i. tdTomatopos cells express higher levels of Bcl11a than tdTomato $^{\text {neg }}$ cells (Figure 1D), indicating specific labelling. At this time point we would expect labelling of progenitor as well as differentiated, Bc/11a-expressing cells in the mammary gland, as previously reported ${ }^{12}$. At 5 days p.i., Bcl11a expression in tdTomatopos cells is reduced compared to 1 day p.i., reflecting the persistence of the progenitors cells labelling after recycling of the mammary gland and loss of the Bc/11a-expressing differentiated cells after 5 days p.i. (Figure 1D).

To confirm the identity of labelled cells, labelling was induced at puberty or in the adult with either 1 single intraperitoneal injection or 3 consecutive days of tamoxifen (1 $\mathrm{mg}$ per injection). Mammary tissues were collected at 1 day p.i. and fluorescence-activated cell sorting (FACS) analysis of the mammary compartments was performed (Figure 2A). One day post-labelling revealed that the vast majority $(95 \% \pm 1.6)$ of tdTomato $^{\text {pos }}$ cells reside within the luminal compartment (Figure 2B, C) and are primarily luminal progenitors (Figure 
104 2D). Interestingly, the contribution of tdTomatopos cells to the luminal compartment increased 8 weeks after labelling (Figure 2E), further confirming the progenitor identity of the labelled Bcl11a luminal cells. To further assess the progenitor nature of $B c / 11 a-$ expressing cells, females were mated 8 weeks after tamoxifen injection and tissues were harvested at gestation day 14.5. We found that the number of tdTomatopos luminal cells expanded significantly to constitute approximately $30 \%$ of the luminal compartment, showing that $B c / 11 a$-expressing progenitor cells contribute to the alveologenesis process (Figure 2F).

To gain insights into the spatial distribution of the labelled cells in situ, we performed optical

114 tissue clearing of whole mammary glands followed by multicolour confocal 3D imaging ${ }^{16,17}$.

115 By visualising the epithelium using EpCAM and cytokeratin-5 (K5), we observed the presence of individual cells and small clusters of tdTomato ${ }^{\text {pos }}$ cells throughout the luminal layer of the mammary gland (Figure 3A). To confirm Bcl11a-labelled cells contribution to the alveologenesis process we analysed the mice at 14.5 days gestation, where we could observe clusters of red cells in the mammary alveoli (Figure 3B).

\section{Conclusion}

122 Here we report the generation of a Bcl11a lineage tracing mouse model. This novel mouse 123 model adds an important tool to study the biology of Bc/11a in health and disease. We 124 validated this new lineage tracing mouse model by assessing labelling in mammary 125 epithelial cells. The reporter mouse recapitulated previous results which report the expression of $B c / 11 a$ in luminal progenitor cells ${ }^{12}$. In addition, our analysis suggest that Bcl11a labelled cells are long-lived and contribute to pregnancy induced alveolar 128 differentiation. The implication of this finding on the role of Bcl11a in tumour initiation 
remains to be explored. In conclusion, this new mouse model provides a powerful tool to explore the role of $\mathrm{Bcl11a}$ in normal development and cancer in the multiple tissues it has been proposed to play a key role.

\section{Figure Legends}

Figure 1. Generation and characterisation of Bcl11a ${ }^{C r e E R T 2}$ mice by lineage tracing.

A, schematic representation of the genetic strategy to insert the CreERT2 cassette into the

3' UTR of the Bcl11a locus. B, long range PCR detecting the 5' and 3' homology arms (5' HA and 3' HA, respectively) of tissue samples from mice with the successful integration of the targeting cassette. The primers used in the long-range PCR are represented as arrows in the schematic in A. C, Schematic describing Bc/11a lineage tracing model. D, To induce Bc/11a tracing, mice were injected intraperitoneally with 3 low doses of tamoxifen on 3 consecutive days post puberty and tissues collected at 1 day ( 1 day p.i.) or 5 days (5 days

p.i.) post injection. Epithelial cells were FACS sorted based on EpCAM expression and RNA extracted. The graph shows the levels of Bcl11a expression normalised to Gapdh in tdTomatopos $^{\text {compared to tdTomato }}{ }^{\text {neg }}$ cells from the same sample, as detected by qRTPCR. Data are represented as mean \pm SD of 3 technical repeats.

Figure 2. Bcl11a labels long-lived luminal progenitors that expand upon gestation.

A, Representative plots showing the gating strategy used to select live, lineage negative, single tdTomato ${ }^{\text {pos }}$ cells in the mouse luminal and basal mammary epithelium, based on EpCAM and Cd49f staining of single cell preparations from lymph node divested mammary glands. FSC-W: forward scatter width, FSC-A: forward scatter area, SSC-A: side scatter area. Luminal differentiated and progenitor cells were identified based on Cd49b and Sca1 staining (Cd49b', differentiated; Cd49b+ Sca1+ and Cd49b+ Sca1', luminal progenitors). B, 
154 Bc/11a labelling was induced by intraperitoneal injection with 1 or 3 low doses of tamoxifen

155 during or post puberty and tissues were collected at 1 day ( 1 d) and 8 weeks (8 w) post 156 labelling and 14.5 days gestation (14.5 dG). The graph shows the distribution of 157 tdTomato ${ }^{\text {pos }}$ cells within the epithelial compartment, analysed by FACS. Data are shown 158 as mean percentage \pm SD. $\mathbf{C}$, to induce Bcl11a tracing, mice were injected as in $\mathbf{B}$ and tissues were collected 1 day post labelling. The graph shows the percentage of tdTomatopos cells in the luminal and basal compartment as determined by FACS. Data are represented as mean \pm SD. $\mathbf{D}$, the graph shows the percentage of tdTomato ${ }^{\text {pos }}$ cells in differentiated $\left(\mathrm{Cd} 49 b^{-}\right)$or progenitor $\left(\mathrm{Cd}_{49 b^{+}}, \mathrm{Sca1}^{+}\right.$or Sca1 $\left.{ }^{-}\right)$luminal cells as determined by FACS 1 day post labelling. E, The graph shows the percentage of tdTomatopos cells in the luminal and basal compartment as determined by FACS 8 weeks $(8 \mathrm{w})$ post labelling. Data are represented as mean \pm SD. F, Bcl11a labelling was induced and mammary glands were collected at 14.5 days gestation ( $14.5 \mathrm{dG})$. The graph shows the percentage of cells in the luminal and basal compartment, as determined by FACS. Data are represented as mean $168 \pm \mathrm{SD}$.

\section{Figure 3. In situ localization of Bcl11a labelled cells in the mammary gland.}

A, Bcl11a tracing was induced as in Figure 2 and tissues were collected at 8 weeks (8 w)

171 post labelling. Confocal 3D imaging of tdTomato ${ }^{\text {pos }}$ cells of CUBIC-cleared whole glands

172 immunostained with K5 and EpCAM are shown as overview; scale bars, $100 \mu \mathrm{m}$. B, Bc/11a 173 labelling was induced and mammary glands were collected at 14.5 days gestation (14.5 $174 \mathrm{dG})$. Confocal 3D imaging of tdTomatopos cells of CUBIC-cleared whole glands 175 immunostained with K5 and EpCAM are shown as overview; scale bars, $100 \mu \mathrm{m}$. 
178 S.P. conceptualised and performed all the experiments. The $B c / 11 a^{C r e E R T 2}$ mice were made

179 by W.T.K. whilst in P.L.'s laboratory. S.P. and W.T.K. wrote the manuscript with input from

180 the other authors. W.T.K. conceptualised and supervised the study.

182 Acknowledgements

183 We would like to thank the staff at Sanger Institute, Research Service Facility (RSF) and 184 the staff at the Cambridge NIHR BRC Cell Phenotyping Hub for their constant support and assistance. W.T.K. is funded by a CRUK Career Establishment Award (C47525/A17348), CRUK Small Molecule Drug Discovery Project Award (C47525/A25850), Breast Cancer Now Project Grant (2017MayPR907), University of Cambridge and Magdalene College, 188 Cambridge.

\section{Materials and Methods}

\section{Mouse experiments}

192 All experimental animal work was performed in accordance to the Animals (Scientific 193 Procedures) Act 1986, UK and approved by the Ethics Committee at the Sanger Institute.

194 Correct targeting was confirmed by long- range PCR. All the primers used for genotyping 195 are listed in Table S1. Details of the lineage tracing experiments are summarised in Table 196 S2. The Rosa26-LSL-tdTomato mouse line was obtained from the Jackson Laboratory 197 (JAX 007905) (Ref 25?). For each experiment, the animals used were heterozygous for $198 B c / 11 a^{C r e E R T 2}$ and homozygous for Rosa26-LSL-tdTomato. Lineage tracing was induced at 199 puberty (5 weeks of age) or in the adult ( 9 weeks of age) with either 1 single intraperitoneal 200 injection or 3 injections on 3 consecutive days of tamoxifen ( $1 \mathrm{mg}$ per injection) in corn oil. $201 \mathrm{~F}$ or each time point $B c / 11 a^{C r e E R T 2}$ wildtype control littermates were injected at the same 
time as experimental animals and tissues collected to set background levels of tdTomato under a 12:12 h light-dark cycle, with water and food available ad libitum and euthanized by terminal anaesthesia.

\section{Mammary gland dissociation into single-cell suspension}

Lymph node divested mammary glands (excluding the cervical pair) were dissected from

the mice and digested O/N in DMEM/F12 (Gibco) $+10 \mathrm{mM} \mathrm{HEPES} \mathrm{(Gibco)}+1 \mathrm{mg} \mathrm{ml}^{-1}$ collagenase (Roche) $+100 \mathrm{U} \mathrm{ml}^{-1}$ hyaluronidase (Sigma) $(\mathrm{CH})+$ gentamicin (Gibco) at $37^{\circ} \mathrm{C}$. After the lysis of red blood cells in $\mathrm{NH}_{4} \mathrm{Cl}$, cells were briefly digested with warm and filtered through a cell strainer (BD Biosciences).

\section{Cell labelling followed by flow cytometry and sorting}

Single-cell suspensions were incubated in HF medium (Hank's balanced salt solution $($ Gibco $)+1 \%$ fetal bovine serum, Gibco) $+10 \%$ normal rat serum (Sigma) for 20 min on ice on ice in HF media. Mouse mammary cells were stained with the following primary antibodies: Cd31-biotin (eBioscience, clone 390, $1 \mu \mathrm{g} \mathrm{m}^{-1}, \quad$ 1:500); Cd45-biotin (eBioscience, clone 30F11, $\left.1 \mathrm{\mu g} \mathrm{ml}^{-1}, 1: 500\right)$; Ter119-biotin (eBioscience, clone Ter119, $\mu \mathrm{gl}^{-1}, 1: 500$ ) and Sca1-AF647 (Biolegend, clone D7, $\left.1 \mathrm{\mu g} \mathrm{ml}^{-1}, 1: 500\right)$. Cells were then stained with streptavidin-APC or Streptavidin-PE/Cy7 (BD-Biosciences, $0.4 \mu \mathrm{g} \mathrm{ml}^{-1}, 1: 500$ ). 
225 7-AAD (Sigma, $10 \mu \mathrm{g} \mathrm{ml}^{-1}, 1: 100$ ) or Zombie Aqua (Biolegend, 1:100) were used to detect

226 dead cells. Cells were filtered through a cell strainer (Partec) before sorting. Sorting of cells

227 was done using a SH800Z sorter (SONY) after sorting calibration was performed with 228 automatic setup beads (SONY) immediately prior to sorting, FACS analysis was performed 229 on a FACS Aria Fusion. Single-stained control cells were used to perform compensation manually. Unstained cells and control animals were used to set gates. The gating strategies is reported in Figure 2A. Cells were sorted for RNA analysis or analysed for tdTomato expression in mammary luminal or basal compartments. FlowJo was used to analyse FACS data.

\section{Optical tissue clearing and wholemount immunostaining}

Mammary tissue was dissected and cut into large pieces $(\sim 15 \times 15 \times 2 \mathrm{~mm})$ for immunostaining and clearing. CUBIC-based tissue clearing ${ }^{18}$ was performed as previously described 16,17 and combined with wholemount immunolabelling for visualization of tdTomato cells. Briefly, CUBIC Reagent 1A was prepared using urea (Sigma, 10\% (w/w)),

N,N,N',N'-tetrakis(2-hydroxypropyl)ethylenediamine (Sigma, 5\% (w/w)), triton-X100 (VWR, $10 \%(\mathrm{w} / \mathrm{w}))$ and $\mathrm{NaCl}($ Sigma, $25 \mathrm{mM})$ in distilled water. CUBIC Reagent 2 was prepared (Sigma, 9\% w/w) and Triton X-100 (VWR, 0.1\% w/w) in distilled water. Tissues were immersed in CUBIC Reagent $1 \mathrm{~A}$ at $37^{\circ} \mathrm{C}$ for 2-3 days. For immunostaining, samples were washed and subsequently blocked in PBS containing triton-X100 (0.5\% (w/v)) and goat serum $(10 \%(\mathrm{v} / \mathrm{v}))$ overnight at $4{ }^{\circ} \mathrm{C}$. Primary antibodies were diluted in blocking buffer at

Fluor conjugated secondary antibodies for 2 days, washed in PBS and transferred to 
249 immersed in CUBIC Reagent 2 for imaging and were imaged within 2 weeks. The following

250 primary antibodies were used for immunostaining: rat anti-EpCAM-AF647 (Biolegend,

251 clone G8.8, $1 \mathrm{\mu g} \mathrm{ml}^{-1}$, 1:250); rabbit anti-K5 (Covance, PRB160P, 1:100). The following

252 Alexa Fluor-conjugated secondary antibodies were purchased from Thermo Fisher

253 Scientific and used 1:500: goat anti-mouse 488 (A11001); goat anti-rat 647 (A21247).

\section{Confocal microscopy and image analysis}

255 Images of wholemount mammary glands were acquired using a Leica TCS SP8 and SP5

256 inverted confocal microscopes with a $40 \times / 1.3 \mathrm{HC}$ PL APO objective lens. Laser power,

257 line averaging and step increment were adjusted manually to give optimal fluorescence 258 intensity for each fluorophore with minimal photobleaching. Image overview and 3D 259 reconstructions were generated using ImageJ software.

260

\section{Preparation of RNA}

262 Sorted cells were spun down and resuspended in RLT, and RNA was extracted using the 263 RNeasy mini kit (for mouse cells) according to manufacturer instructions. DNA was 264 degraded by adding 20U Rnase-free Dnasel (Roche) for $30 \mathrm{~min}$ at room temperature. 265 Dnasel treatment was performed on columns.

267 Preparation of cDNA and qRT-PCR

268 Total RNA was diluted to a final volume of $11 \mu \mathrm{l} .2 \mu \mathrm{l}$ of random primers (Promega) were 269 added after which the mixture was incubated at $65^{\circ} \mathrm{C}$ for $5 \mathrm{~min}$. A master mix containing

270 Transcriptor Reverse Transcriptase (Roche), Reverse Transcriptase buffer, 2 mM dNTP 271 mix and RNasin Ribonuclease Inhibitors (Promega). This mixture was incubated at $25^{\circ} \mathrm{C}$ 272 for $10 \mathrm{~min}$, then $42^{\circ} \mathrm{C}$ for $40 \mathrm{~min}$ and finally $70^{\circ} \mathrm{C}$ for $10 \mathrm{~min}$. The resulting cDNA was then 
273 diluted 1:2.5 in $\mathrm{H}_{2} \mathrm{O}$ for subsequent use. qPCR was performed using a Step-One Plus Real-

274 Time PCR System (Thermofisher Scientific). Taqman (ThermoFisher Scientific) probes

275 with GoTaq Real Time qPCR Master Mix (Promega) were used. The enrichment was

276 normalised with control mRNA levels of GAPDH and relative mRNA levels were calculated

277 using the $\Delta \Delta \mathrm{Ct}$ method comparing to control group. For the list of primers and probes see

278 Table S1.

279

280

281

282

283

284

285

286

287

\section{References}

1. Yin, J., Xie, X., Ye, Y., Wang, L. \& Che, F. BCL11A: a potential diagnostic biomarker and therapeutic target in human diseases. Biosci. Rep. 39, BSR20190604 (2019).

2. Nakamura, T. et al. Evi9 encodes a novel zinc finger protein that physically interacts with BCL6, a known human B-cell proto-oncogene product. Mol. Cell. Biol. 20, 3178-3186 (2000).

3. Masliah-Planchon, J., Bièche, I., Guinebretière, J.-M., Bourdeaut, F. \& Delattre, O. SWI/SNF Chromatin Remodeling and Human Malignancies. Annu. Rev. Pathol. Mech. Dis. 10, 145-171 (2015).

4. Tang, L., Nogales, E. \& Ciferri, C. Structure and Function of SWI/SNF Chromatin Remodeling Complexes and Mechanistic Implications for Transcription. Prog. Biophys. Mol. Biol. 102, 122-128 (2010).

5. Liu, P. et al. Bcl11a is essential for normal lymphoid development. Nat. Immunol. 4, 525-532 (2003).

6. $\mathrm{Yu}, \mathrm{Y}$. et al. Bcl11a is essential for lymphoid development and negatively regulates p53. J. Exp. Med. 209, 2467-2483 (2012).

7. Sankaran, V. G. et al. Developmental and species-divergent globin switching are driven by BCL11A. Nature 460, 1093-1097 (2009).

8. Li, S. et al. Transcription Factor CTIP1/ BCL11A Regulates Epidermal Differentiation and Lipid Metabolism During Skin Development. Sci. Rep. 7, 13427 (2017). 
305 9. Ippolito, G. C. et al. Dendritic cell fate is determined by BCL11A. Proc. Natl. Acad. Sci. U. S. A. 111, (2014).

10. Xu, J. et al. Correction of Sickle Cell Disease in Adult Mice by Interference with Fetal Hemoglobin Silencing. Science (80-. ). 334, 993 LP - 996 (2011).

11. Esrick, E. B. et al. Post-Transcriptional Genetic Silencing of BCL11A to Treat Sickle Cell Disease . N. Engl. J. Med. 384, 205-215 (2021).

12. Khaled, W. T. et al. ARTICLE BCL11A is a triple-negative breast cancer gene with critical functions in stem and progenitor cells. Nat. Commun. 6, (2015).

13. Lazarus, K. A. et al. BCL11A interacts with SOX2 to control the expression of epigenetic regulators in lung squamous carcinoma. Nat. Commun. 9, 1-11 (2018).

14. Bass, A. J. et al. SOX2 is an amplified lineage-survival oncogene in lung and esophageal squamous cell carcinomas. Nat Genet 41, 1238-1242 (2009).

15. Kretzschmar, K. \& Watt, F. M. Lineage tracing. Cell 148, 33-45 (2012).

16. Lloyd-Lewis, B. et al. Imaging the mammary gland and mammary tumours in 3D: optical tissue clearing and immunofluorescence methods. Breast Cancer Res. 18, 127 (2016).

17. Davis, F. M. et al. Single-cell lineage tracing in the mammary gland reveals stochastic clonal dispersion of stem/progenitor cell progeny. Nat Commun 7, 13053 (2016).

18. Susaki, E. A. et al. Whole-brain imaging with single-cell resolution using chemical cocktails and computational analysis. Cell (2014) doi:10.1016/j.cell.2014.03.042. 
Pensa S. et al.

A
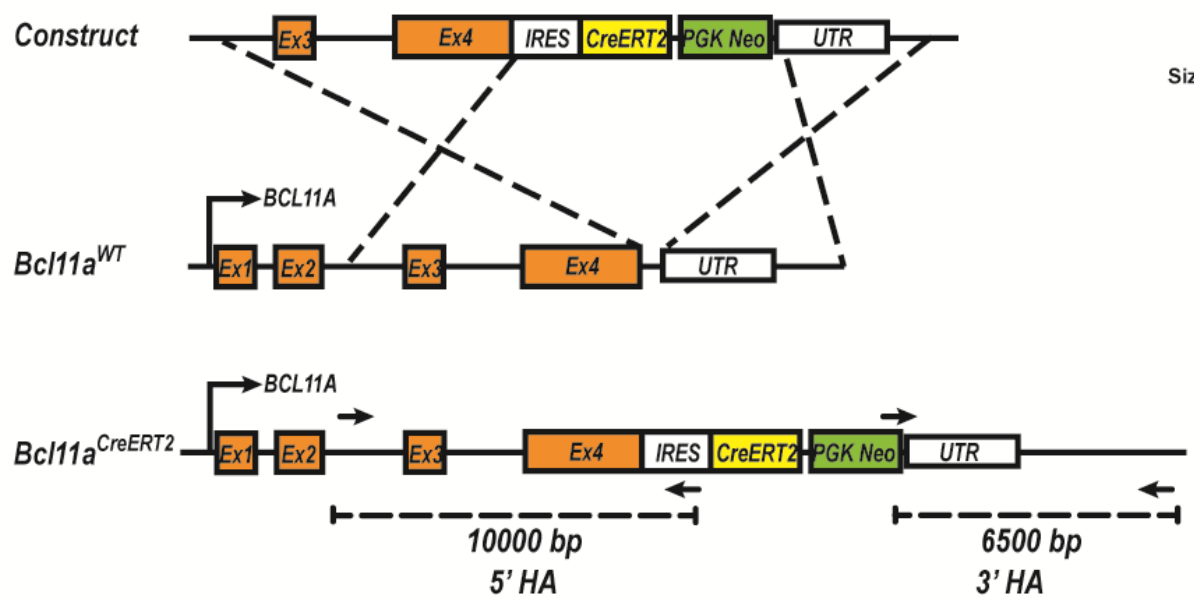

C

\section{Bcl11a Lineage tracing mouse model}

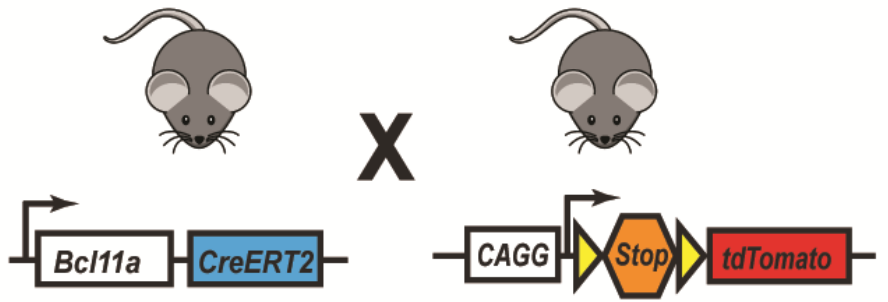

Figure 1

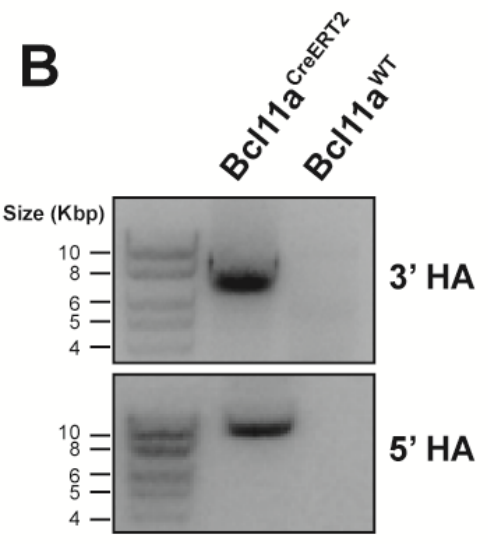

D
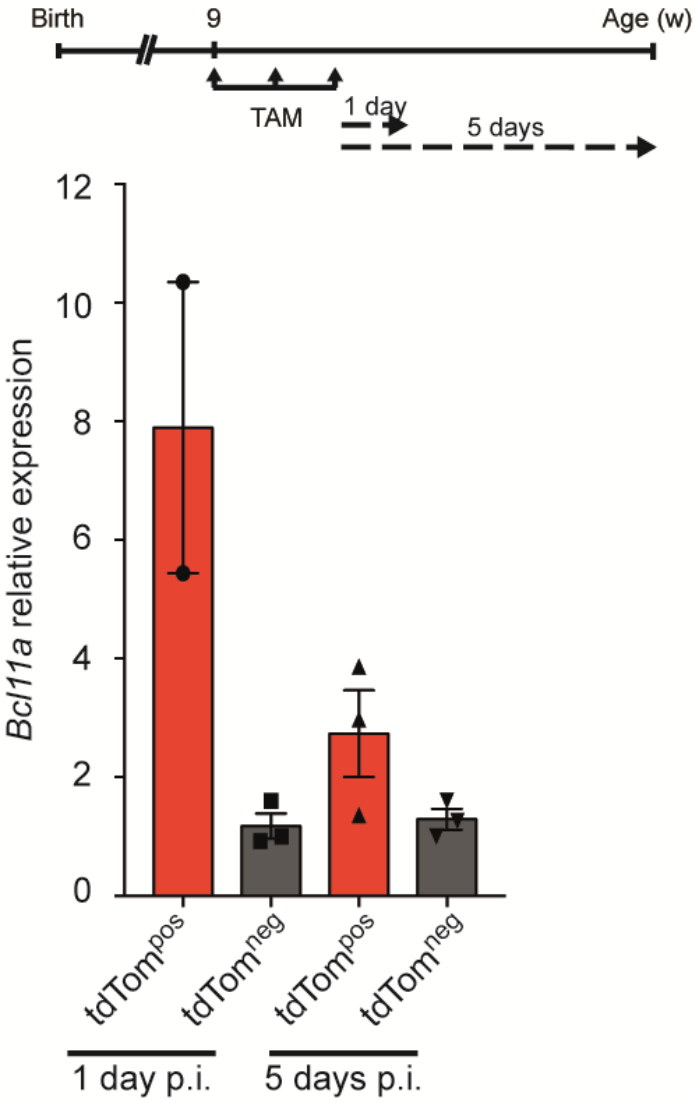


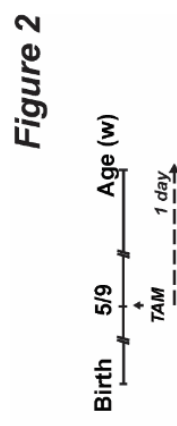

口
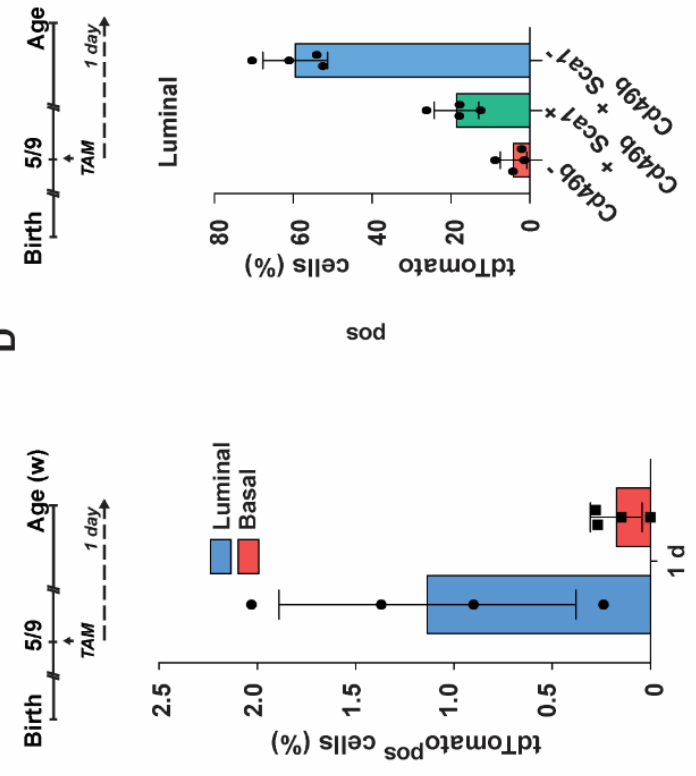

sod

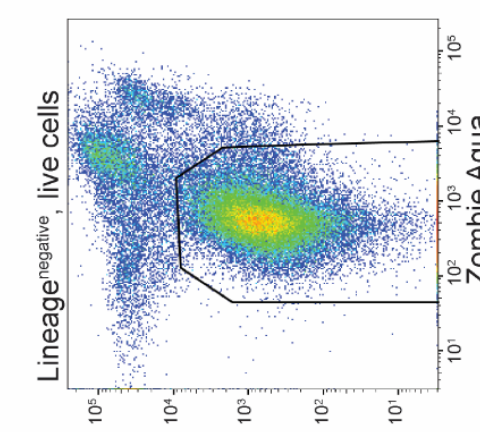

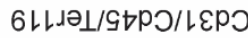
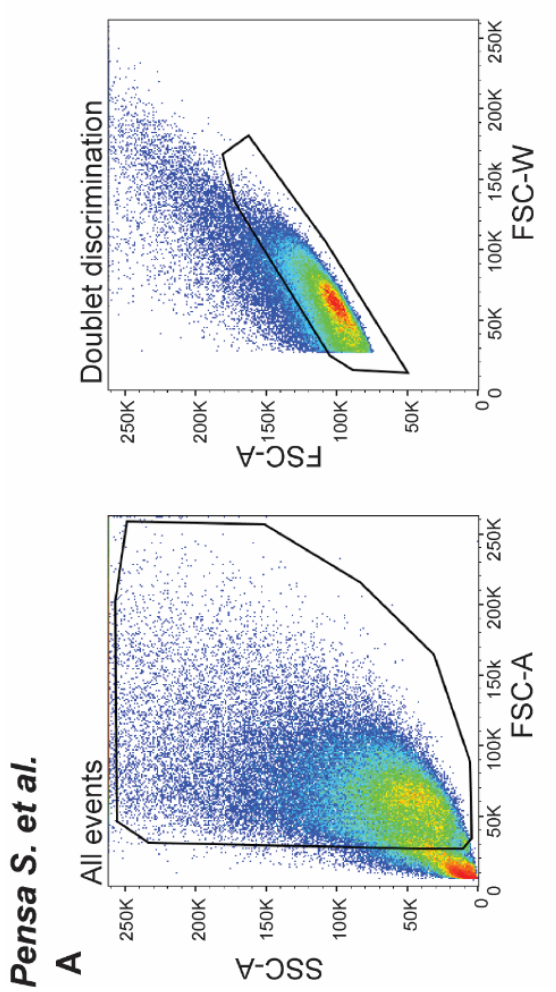
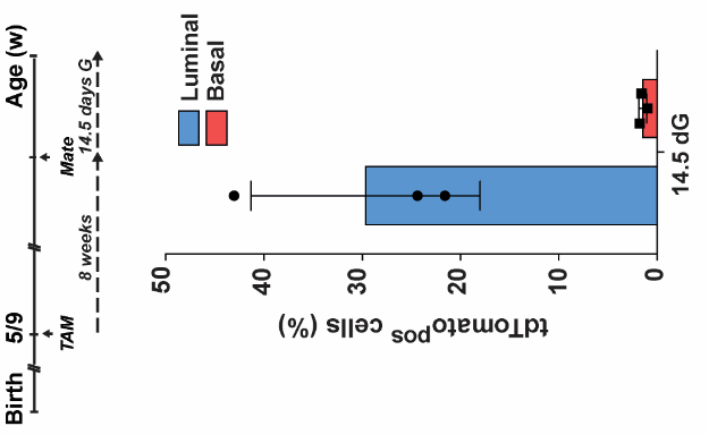

น
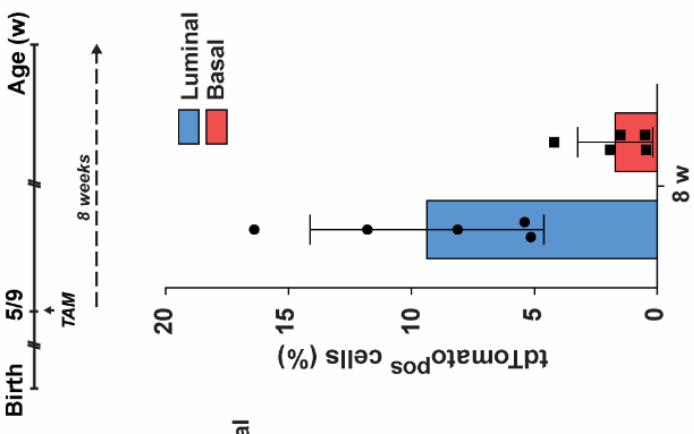

ш

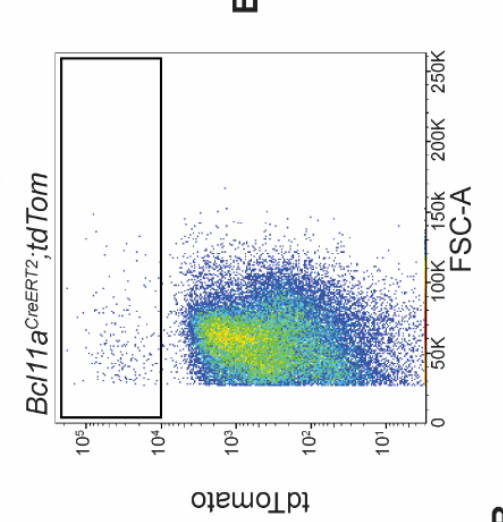

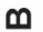
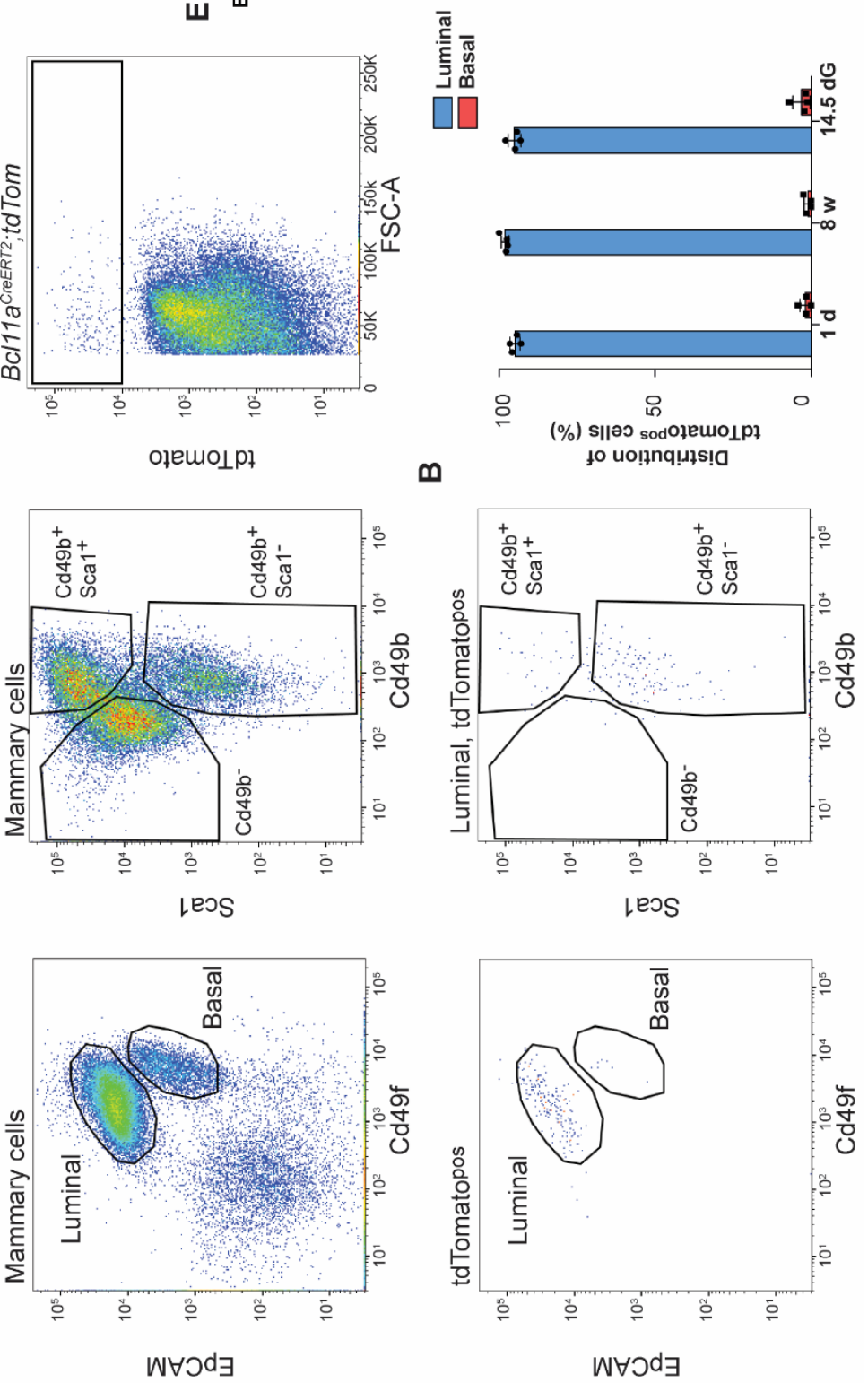
ำ
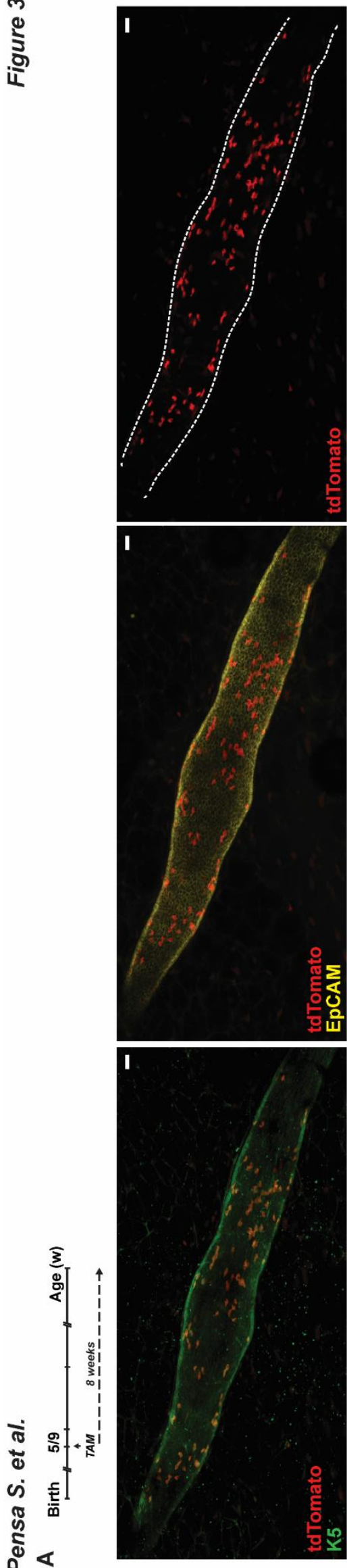
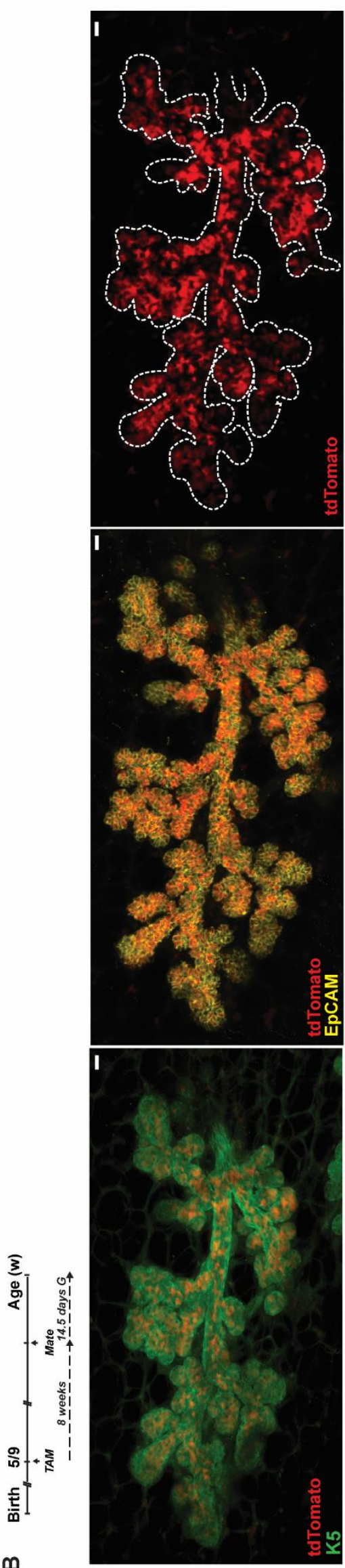


\begin{tabular}{|l|l|l|}
\hline $\begin{array}{l}\text { Table S1 - Genotyping } \\
\text { primers list and qRT-PCR } \\
\text { probes list }\end{array}$ & & \\
\hline & & \\
\hline Gene & Forward Primer (5' - 3') & Size of PCR products (bp) \\
\hline CreERT-long range-5p-FW & GAGACAAGAACAGGTGCAAGAGTGGATT & 10000 \\
\hline CreERT-long range-5p-RV & GGGGGTTCCGCTGCCTG & \\
\hline CreERT-long range-3p-FW & CCCTGCAGCAGCAGCACCAG & 6500 \\
\hline CreERT-long range-3p-RV & CAGCGAGGTCCCCTTTCTCACTAAAAAT & \\
\hline CreERT-FW & ATTTGTAAGATGCCTTTTAGCGTG & 187 (Mut) \\
\hline CreERT-Mut-RV & GGGGGTTCCGCTGCCTG & 585 (wild type) \\
\hline CreERT-WT-RV & GCGTTGTTTTCTACCCTCCC & \\
\hline R26-tdTomato-WT-FW & AAGGGAGCTGCAGTGGAGTA & 297 (wild type) \\
\hline R26-tdTomato-WT-RV & CCGAAAATCTGTGGGAAGTC & 196 (Mut) \\
\hline R26-tdTomato-Mut-FW & GGCATTAAAGCAGCGTATCC & \\
\hline R26-tdTomato-Mut-RV & CTGTTCCTGTACGGCATGG & \\
\hline Gene & Taqman Probe details & Company \\
\hline Bcl11a & Mm00479358_m1 & Life Technologies \\
\hline Gapdh & Mm99999915_g1 & Life Technologies \\
\hline
\end{tabular}

\begin{tabular}{|l|c|}
\hline Table S2 - Lineage tracing data & \\
\hline & \\
\hline Chase group, 1 TAM injection & n of animals \\
\hline 1 day & 4 \\
\hline 5 days & 3 \\
\hline & \\
\hline & \\
\hline & $\mathbf{n}$ of animals \\
\hline Chase group, 3 TAM injection & 1 \\
\hline 1 day & 1 \\
\hline 5 days & 5 \\
\hline 8 weeks & 3 \\
\hline 14.5 days gestation & \\
\hline
\end{tabular}

